\title{
Transcript profiling of the immunological interactions between Actinobacillus pleuropneumoniae serotype 7 and the host by dual RNA-seq
}

Ping $\mathrm{Li}^{1,2+} \mathrm{D}$, Zhiwen $\mathrm{Xu}^{1,2+}$, Xiangang Sun ${ }^{1}$, Yue Yin ${ }^{1,2}, \mathrm{Yi} \mathrm{Fan}^{1,2}$, Jun Zhao ${ }^{1,2}$, Xiyu Mao ${ }^{1,2}$, Jianbo Huang ${ }^{1,2}$, Fan Yang ${ }^{1,2}$ and Ling Zhu $u^{1,2^{*}}$

\begin{abstract}
Background: The complexity of the pathogenic mechanism underlying the host immune response to Actinobacillus pleuropneumonia (App) makes the use of preventive measures difficult, and a more global view of the host-pathogen interactions and new insights into this process are urgently needed to reveal the pathogenic and immune mechanisms underlying App infection. Here, we infected specific pathogen-free Mus musculus with App serotype 7 by intranasal inoculation to construct an acute hemorrhagic pneumonia infection model and isolated the infected lungs for analysis of the interactions by dual RNA-seq.

Results: Four cDNA libraries were constructed, and 2428 differentially expressed genes (DEGs) of the host and 333 DEGs of App were detected. The host DEGs were mainly enriched in inflammatory signaling pathways, such as the TLR, $\mathrm{NLR}, \mathrm{RLR}, \mathrm{BCR}$ and TCR signaling pathways, resulting in large-scale cytokine up-regulation and thereby yielding a cytokine cascade for anti-infection and lung damage. The majority of the up-regulated cytokines are involved in the IL-23/IL-17 cytokine-regulated network, which is crucial for host defense against bacterial infection. The DEGs of App were mainly related to the transport and metabolism of energy and materials. Most of these genes are metabolic genes involved in anaerobic metabolism and important for challenging the host and adapting to the anaerobic stress conditions observed in acute hemorrhagic pneumonia. Some of these genes, such as adhE, dms A, and aspA, might be potential virulence genes. In addition, the up-regulation of genes associated with peptidoglycan and urease synthesis and the restriction of major virulence genes might be immune evasion strategies of App. The regulation of metabolic genes and major virulence genes indicate that the dominant antigens might differ during the infection process and that vaccines based on these antigens might allow establishment of a precise and targeted immune response during the early phase of infection.
\end{abstract}

Conclusion: Through an analysis of transcriptional data by dual RNA-seq, our study presents a novel global view of the interactions of App with its host and provides a basis for further study.

Keywords: A. Pleuropneumoniae, Dual RNA-seq, Immunological interactions, Immune response, Anaerobic metabolism, Immune evasion

\footnotetext{
* Correspondence: abtczl72@126.com

${ }^{\dagger}$ Equal contributors

${ }^{1}$ College of Veterinary Medicine, Sichuan Agricultural University, Huimin Road

211, Weenjiang District, Chengdu, Sichuan, China

${ }^{2}$ Key Laboratory of Animal Diseases and Human Health of Sichuan Province,

Chengdu, Sichuan, China
} 


\section{Background}

Actinobacillus pleuropneumonia (App) can cause serious porcine respiratory diseases with high incidence and mortality rates. Fifteen serotypes of App have been detected, and these can cause acute hemorrhagic pneumonia and chronic interstitial pneumonia and have markedly affected the porcine industry [1]. Even with the optimization of intensive and large-scale breeding patterns and the development of vaccines for the disease, many districts have observed high morbidity rates due to $A p p$ in recent years [2-4]. This disease is airborne, and the wild strains can easily break through the defense of a sound vaccine prevention and control system, resulting in rapid proliferation. Moreover, the infection and pathogenesis of $A p p$ have become increasingly complex, particularly in cases of mixed infection [5]. An extensive body of clinical cases have reported that many wild strains show drug resistance to some routinely used antibiotics, such as tetracyclines [6], penicillins [7], erythromycin, streptomycin and tiamulin [8]. The evolution of drug resistance has resulted in the failure of drug treatment. Therefore, the development of efficient vaccines is an important undertaking, and to detect potential vaccine candidates, many virulence genes have been identified. Researchers have then analyzed the function of these genes, such as $H g b A$ [9], ureC [10] and apxIA [11], through gene knockout to construct gene deletion mutants, providing more choices for subunit vaccine production.

The infection process is complex, and the interactions between a pathogen and its host are essential components of the infection. The pathogenesis and anti-infection mechanisms determine the development of the disease course. In fact, these mechanisms influence and restrict each other and ultimately yield differences in gene expression. Because the internal environment of the host shows marked differences from the in vitro environment, the expression of virulence factors by $A p p$ is also notably different [12]; for example, apxIVA is differentially expressed in the two environments [13]. With the aim of determining the key point of the infection process and the differences between the environments, many experiments based on highthroughput sequencing have been conducted to identify differences in App gene expression between in vivo and in vitro conditions. Deslandes et al. [14] analyzed the differentially expressed genes (DEGs) of $A p p$ during the acute phase of infection by microarray hybridization and identified three outer membrane proteins or lipoproteins, namely fhaB (APL_0959), irp (APL_0919) and APL_0920, as potential candidates for a protective cross-serotype vaccine. Klitgaard et al. [15] used DNA microarrays to analyze the potential strategies used by $A p p$ for survival and persistence in the host. In addition, Zuo et al. [16] analyzed transcription in swine lung tissue by microarray hybridization to assess the regulation of the host defense response. However, the interactions between $A p p$ and the host have not yet been elucidated. Brogaard et al. [17] selected 17 bacterial and 31 host genes from infected lungs and conducted the first high-throughput RT-qPCR analysis of the interactions between App and the host. Nevertheless, these techniques can detect only known genes and do not identify differentially expressed genes for which annotation information is lacking.

Based on these questions, we aimed to investigate the interaction between $A p p$ and the host through RNA-seq. Different from microarray hybridization and high-throughput qPCR, RNA-seq can collect almost all transcription information from a specific sample(s) rather than monitor some specific genes. As a result, this technique can provide a global view of the interactions between the pathogen and the host [18, 19]. Moreover, we assessed transcription information for App and the host separately by dual RNA-seq and screened for potential causal relationships and interactions. In addition, a mouse model was selected for the study of App infection. Because specific pathogen-free (SPF) pigs are rare and costly and pigs in the field are easy affected by the pathogens found in the environment and vaccines, we had to select an animal model that is more easily available for this study. Compared with SD rat, guinea pig and rabbit, the SPF mouse is more susceptible [20], and the pathological changes caused by $A p p$ are similar to those observed in typical cases of pigs naturally infected with App [21]; thus, a mouse model have been widely used for testing medicines and vaccines against App. Therefore, the present study also lays a foundation for the study of App infection in a mouse model and provides supporting data.

\section{Results \\ Observations of symptoms and histopathology}

In the current study, Mus musculus (Mmu) animals infected with App deteriorated over time and showed serious hemorrhagic pulmonary inflammation. Four hours after infection, the infected animals behaved differently from the control group, and these behavioral changes included anepithymia and depression. Six hours after infection, all of the infected animals exhibited depression, polypnea and disordered fur. In addition, 8 and $12 \mathrm{~h}$ after infection, two of the infected animals died, and another animal died $10 \mathrm{~h}$ post infection. In contrast, all of the animals in the control group were healthy and energetic.

A histopathological analysis of the lungs by HE staining showed that all of the infected lungs were acutely inflamed and showed hyperemia, bleeding and infiltration of fibrin and inflammatory cells, including lymphocytes, 
macrophages, neutrophils and some eosinophilic granulocytes (Figs 1b-f). In addition, the infection led to degeneration of the alveolar septal cells and thickening of the interalveolar septa (Fig. 1b-f). These pathological changes were similar to those detected in a pig model by Brogaard et al. [17]. In summary, a hemorrhagic pulmonary inflammation model of App infection through intranasal inoculation was successfully established and used to obtain samples for dual RNA-seq.

\section{Transcriptomes of Actinobacillus pleuropneumoniae and Mus musculus}

A comparison of the App content in all infected lungs detected by qRT-PCR was performed, and the mean $\mathrm{Ct}$ values of the five groups were 27.2, 27.27, 24.05, 28.88 and 29.42, respectively. Accordingly, five RNA samples obtained $8 \mathrm{~h}$ after infection showed a high content of App, with Ct values of 20.86, 22.09, 23.2, 24.56 and 25.61 , and this time point was thus selected for sequencing. In addition, the RNA samples of bacterial cells and five healthy lungs were chosen as control groups for DEG identification.

To construct libraries of the infected lungs, $67.68 \%$ of reads were mapped to the reference genome of $M m u$, and $15.68 \%$ were mapped to $A p p$, which provided sufficient data for further analysis. For the healthy lungs and App libraries, the mapped rates were $85.01 \%$ and $97.99 \%$, respectively (Table 1 ). DEGs (fold change $\geq 2$ and FDR $<0.01$ ) were defined using EB-Seq software (Fig. 2) and were annotated with the COG, GO, KEGG, Swiss-Prot and $\mathrm{nr}$ databases (Table 2). A total of 2428 DEGs were found in the infected lung tissue compared with healthy tissue, and of these, 1484 and 944 genes were up-regulated and down-regulated, respectively. A total of 333 DEGs of App were detected in the infected lungs compared with the in vitro conditions, and of these DEGs, 113 and 220 were up-regulated and downregulated, respectively.

Enrichment analysis of the DEGs found in lung tissue showed that the genes were mainly associated with the

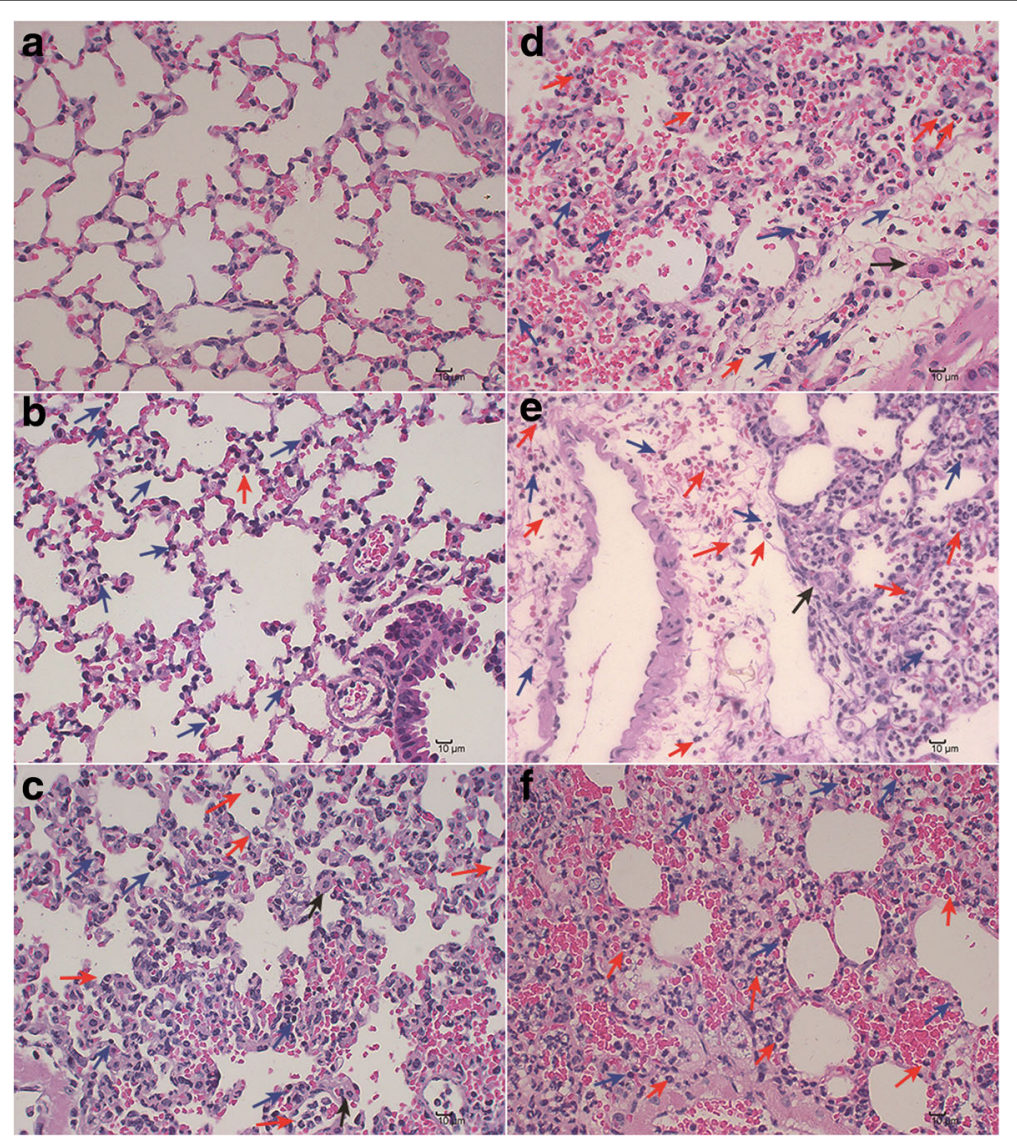

Fig. 1 HE staining of lung tissue. Arrows: blue - leukomonocytes, red - neutrophils, black - macrophages. a Lung tissues from the control group. These tissues show a few RBCs from the trachea and bronchus in the interalveolar septa. Scale bar, $10 \mu \mathrm{m}$. b-f Lung tissues from the treatment groups. Scale bar, $10 \mu \mathrm{m}$. b Four hours after infection, interalveolar septa shows infiltration of inflammatory cells. c Six hours after infection, the interalveolar septa shows an increase of inflammatory cells, bleeding, and thickening. $\mathbf{d}$ Eight hours after infection, the interalveolar septa is filled with fibrin and cells, including leukomonocytes, neutrophils, macrophages and RBCs. e Ten hours after infection, a vein and interalveolar septa exhibit numerous neutrophils. $\mathbf{f}$ Twelve hours after infection, serious thickening of the interalveolar septa, with bleeding and infiltration of neutrophils and leukomonocytes, is observed 
Table 1 RNA sequencing reads of four cDNA libraries

\begin{tabular}{|c|c|c|c|c|}
\hline Sources $^{a}$ & Total Reads $^{\mathrm{b}}$ & Libraries $^{c}$ & Mapping to $A p p^{d}$ & Mapping to $M m u^{\mathrm{e}}$ \\
\hline \multirow[t]{2}{*}{ Infected lungs } & $101,449,062(100.00 \%)$ & T01 & $15,911,177(15.68 \%)$ & \\
\hline & & T03 & & $68,663,015(67.68 \%)$ \\
\hline App & $13,934,718(100.00 \%)$ & T02 & $13,654,587(97.99 \%)$ & \\
\hline Healthy lungs & $74,591,572(100 \%)$ & T04 & & $63,411,879(85.01 \%)$ \\
\hline
\end{tabular}

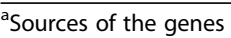

${ }^{\mathrm{b}}$ Quantity of the reads (normalized to $100 \%$ )

"Names of libraries: "T01", genes isolated from infected lungs and mapped to App; "T02", genes isolated from App growing in culture media and mapped to App;

"T03", genes isolated from infected lungs and mapped to Mmu; "T04", genes isolated from healthy lungs and mapped to Mmu

${ }^{\mathrm{d}}$ Quantity of genes mapped to App and percentage of total reads

${ }^{\mathrm{e}} \mathrm{Quantity}$ of genes mapped to $\mathrm{Mmu}$ and percentage of total reads
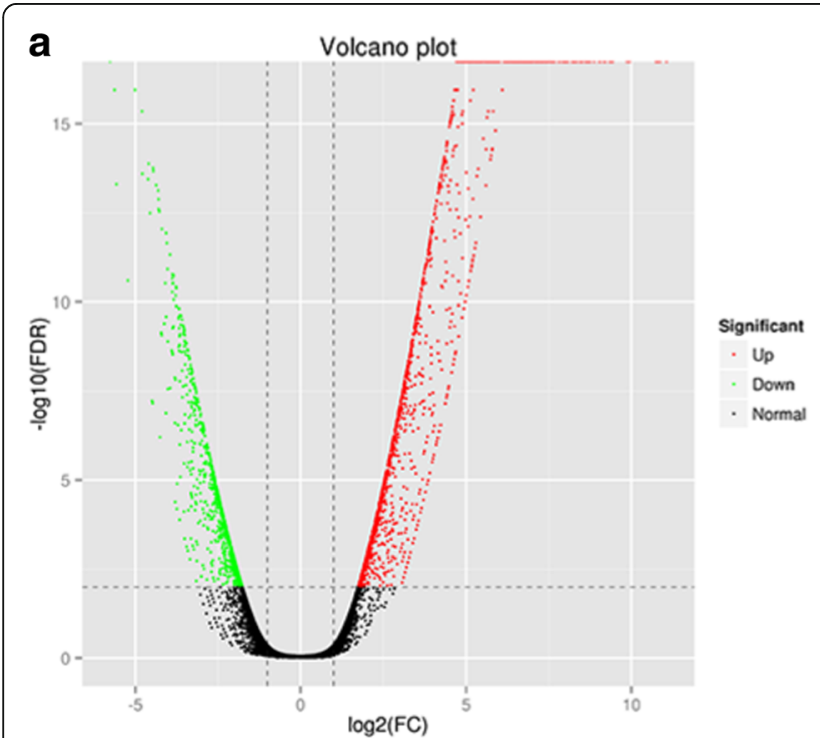

b
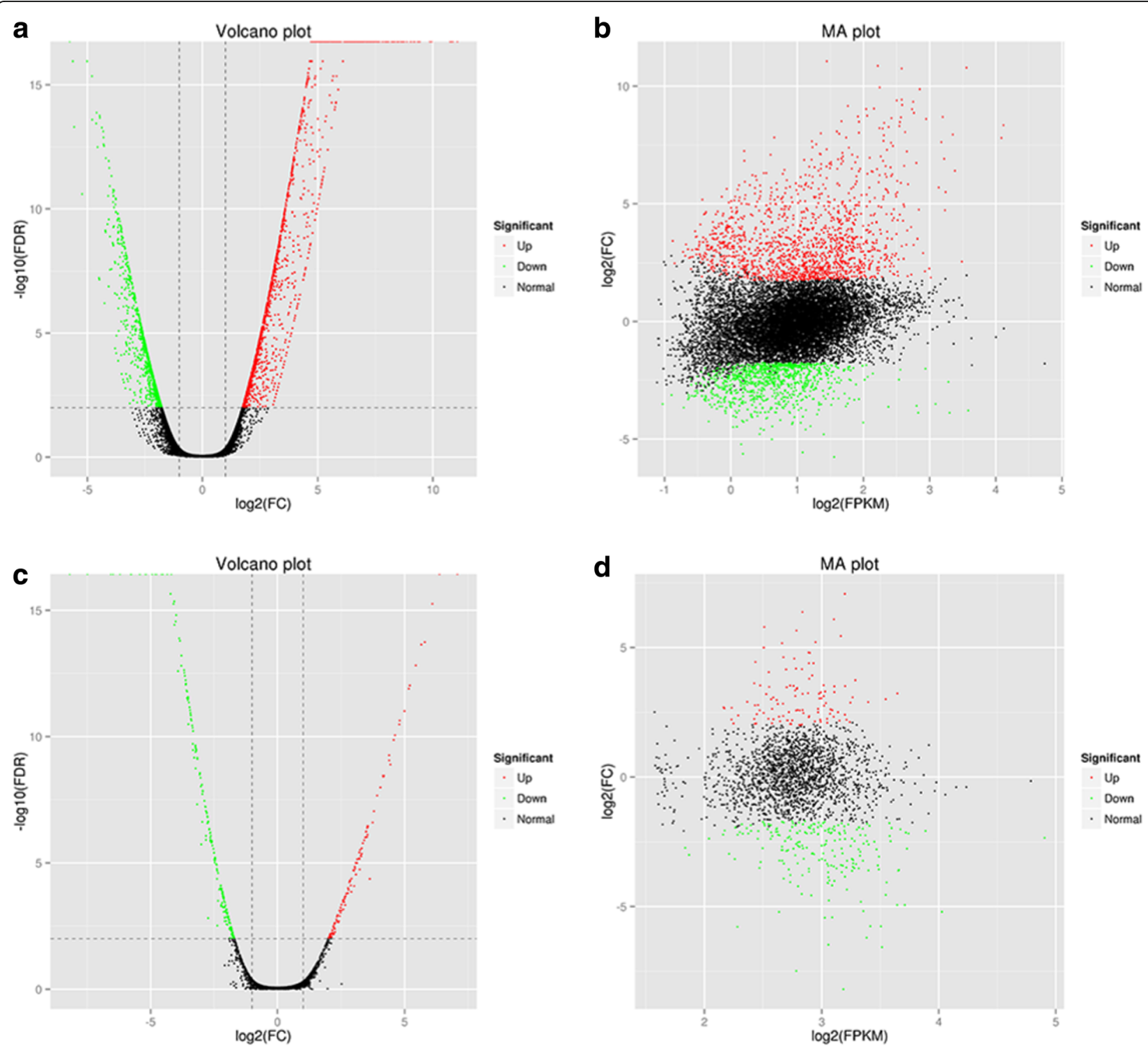

Fig. 2 Overview on differences in gene expression. $\mathbf{a}, \mathbf{b}$ DEGs of Mmu: the $\log _{2}$ (fold change) values of the up-regulated and down-regulated genes were in the intervals of $[1.47,11.04]$ and $[-5.76,-1.75]$, respectively, and the FPKM, which is used to measure the level of transcription or gene expression, was in the interval of $(-4,32)$. $\mathbf{c}$, $\mathbf{d}$ DEGs of App: the $\log _{2}$ (fold change) values of the up-regulated and down-regulated genes were in the intervals of $[2.00,7.07]$ and $[-8.18,-1.70]$, respectively, and the FPKM was in the interval of $(2,32)$ 
Table 2 Statistics of annotated DEGs

\begin{tabular}{lllllll}
\hline DEGs $^{a}$ & \multicolumn{6}{l}{ Annotated databases $^{\mathrm{b}}$} \\
\cline { 2 - 7 } & COG & GO & KEGG & Swiss-Prot & $\mathrm{nr}$ & All \\
\hline Mmu & 650 & 2132 & 1407 & 2224 & 2354 & 2354 \\
App & 267 & 275 & 237 & 293 & 331 & 331
\end{tabular}

${ }^{\mathrm{a} D E G s}$ of $M m u$ and App

bQuantity of DEGs annotated in the Clusters of Orthologous Groups (COG), Gene Ontology (GO), Kyoto Encyclopedia of Genes and Genomes (KEGG),

Swiss-Prot and $\mathrm{nr}$ databases

"signal transduction mechanisms", "replication, recombination and repair", "transcription" and "general function prediction only" terms of the COG database (Fig. 3). To analyze the relationship between these DEGs and the immune system of the host, we annotated these genes according to KEGG pathways for statistics and analysis (Fig. 4). We identified 20 pathways that were enriched and shows statistically significant differences (Fig. 5). Notably, these pathways were mainly related to signal transduction mechanisms.

The DEGs of App were enriched in "energy production and conversion", "inorganic ion transport and metabolism", "amino acid transport and metabolism", "carbohydrate transport and metabolism", and other COG terms (Figs. 3 and 4) and in "carbon metabolism", "fructose and mannose metabolism", "pyruvate metabolism", "amino sugar and nucleotide sugar metabolism", and other KEGG pathways (Fig. 5). These genes represented the in vivo adaptation of the bacterial metabolism to the stresses present in the internal environment. Some metabolic genes also play key roles in pathogenicity.

In addition, 18 genes showing high differences in expression, as identified by RNA sequencing, were selected to validate the gene expression findings obtained by qRT-PCR in order to confirm the reliability of the data. The five RNA samples from infected lungs and the sample used for sequencing were subjected to qRT-PCR assays, and their dispersion was analyzed. A strong correlation $\left(R^{2}=0.989\right)$ in gene expression was found between the RNA-seq and qRT-PCR results (Fig. 6).

\section{Anti-infection mechanism of the host associated with antigen recognition and immune response pathways}

The analysis of the DEGs enriched in the host showed that the infection activated many antigen recognition and response pathways involved in the innate immune response, adaptive immune response and intercellular inflammatory signal transduction and directly showed the up-regulation of the genes of these pathways. In addition, many points of crosstalk were found among these signaling pathways (Additional file 1).
Innate immune response: activation of pattern recognition receptors (PRRs)

Infection by $A p p$ activated the PRR signaling pathways of the host, including the NOD-like receptor (NLR) signaling pathway (ko04621), the Toll-like receptor (TLR) signaling pathway (ko04620), the cytosolic DNA-sensing pathway (ko04623), Fc gamma R-mediated phagocytosis (ko04666), and caused up-regulation of Tnf, Illb, Il6,

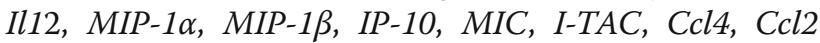
and $C x c l 10$. Interestingly, the RIG-I-like receptor (RLR) signaling pathway (ko04622), which is usually known for its antiviral activity, particularly in response to RNA viruses, was found to be activated in the present study, and caused up-regulation of Tnf, Il12 and IP10 for the adjustment of protein synthesis, growth arrest, dendritic cell activation, NK cell activation, CTL differentiation, antibody production and apoptosis.

Innate immune response: activation of effector molecules and cells

The infection also activated many signaling pathways of effector molecules, including the TNF signaling pathway (ko04668), cytokine-cytokine receptor interaction (ko04060), the chemokine signaling pathway (ko04062) and the phagosome pathway (ko04145). Many cytokines can be regulated by the TNF signaling pathway, and the infection caused up-regulation of genes encoding leukocyte recruitment factors ( $\mathrm{Ccl} 2$, Ccl20, Cxcl1, Cxcl2, Cxcl3 and Cxcl10), leukocyte activation factors (Csf1 and Csf2), surface receptors (Fas), inflammatory cytokines (Il1b, Il6, Il15, Lif and Tnf), intracellular signaling molecules (Bcl3, Nfkbia, Socs3, Tnfaip3 and Traf1), transcription factors (Fos and $J u n B)$, an extracellular matrix remodeling factor (Mmp9), PRRS (Nod2), and cell adhesion factors (Icam1, Sele and Vcam1) and synthesis of inflammatory mediators (Ptgs2). The up-regulation of these proteins resulted in cytokine-cytokine receptor interaction. We determined that the up-regulated genes encoding chemokines included the CXC subfamily (Cxcl9, Cxcl10, Cxcl11 and Cxcl13) and the CC subfamily (Ccl20, Ccl19, Ccl2, Ccl4, Ccl3, Ccl7, Ccl8 and Ccl11), which further activated the chemokine signaling pathway and mediated a wider range of cytokine regulation.

In addition, both FcyR- and ER-mediated phagocytosis in the phagosome pathway (ko04145) were activated by the binding of the pathogen with phagocytosis-promoting receptors. F-actin on the membrane was up-regulated and promoted the internalization and formation of phagosomes with NADPH oxidase. In addition, under the action of the endoplasmic reticulum, the pathogen was degraded by $\mathrm{H}_{2} \mathrm{O}_{2}$ and bound to MHCI and MHCII for processing 


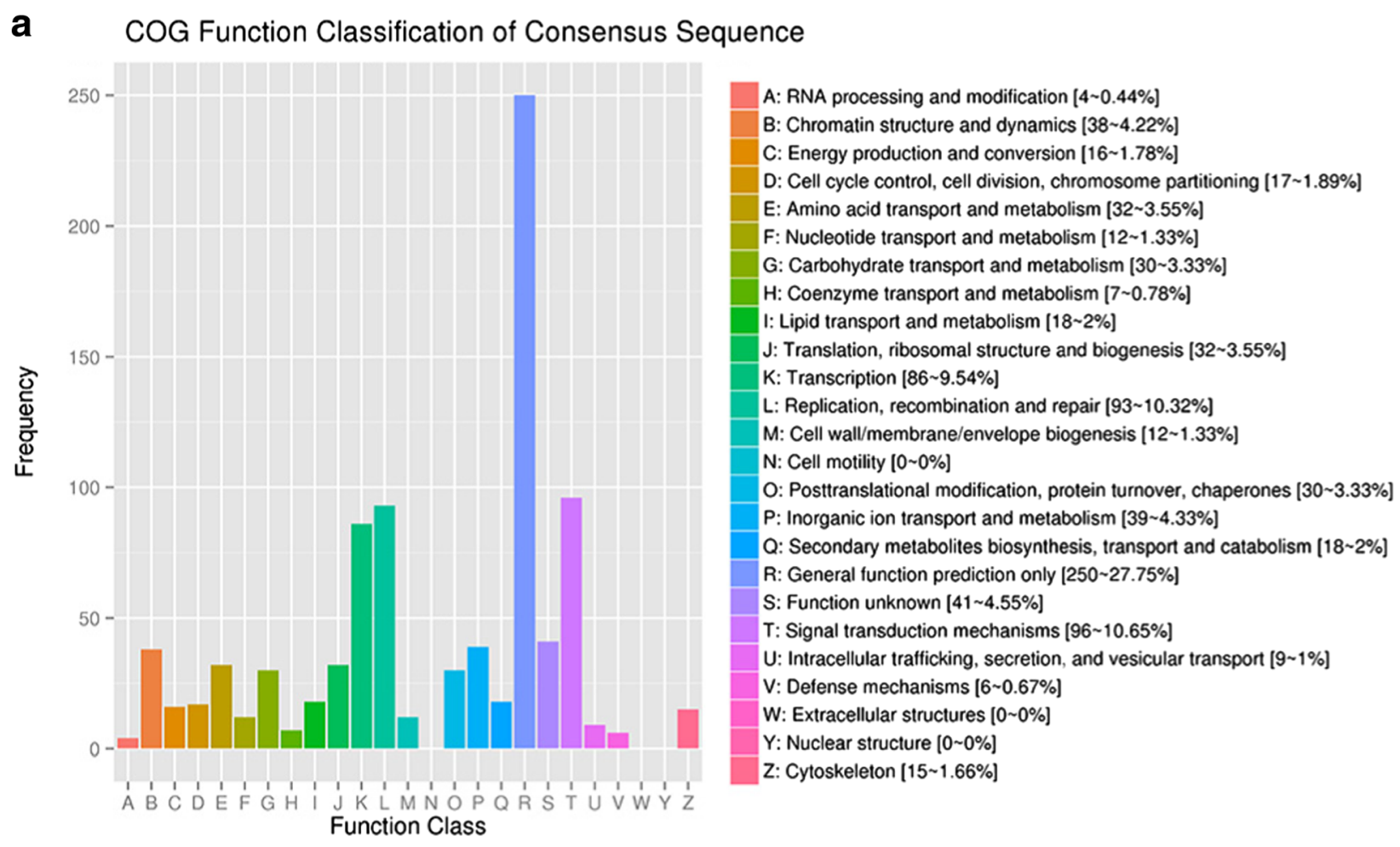

\section{b COG Function Classification of Consensus Sequence}

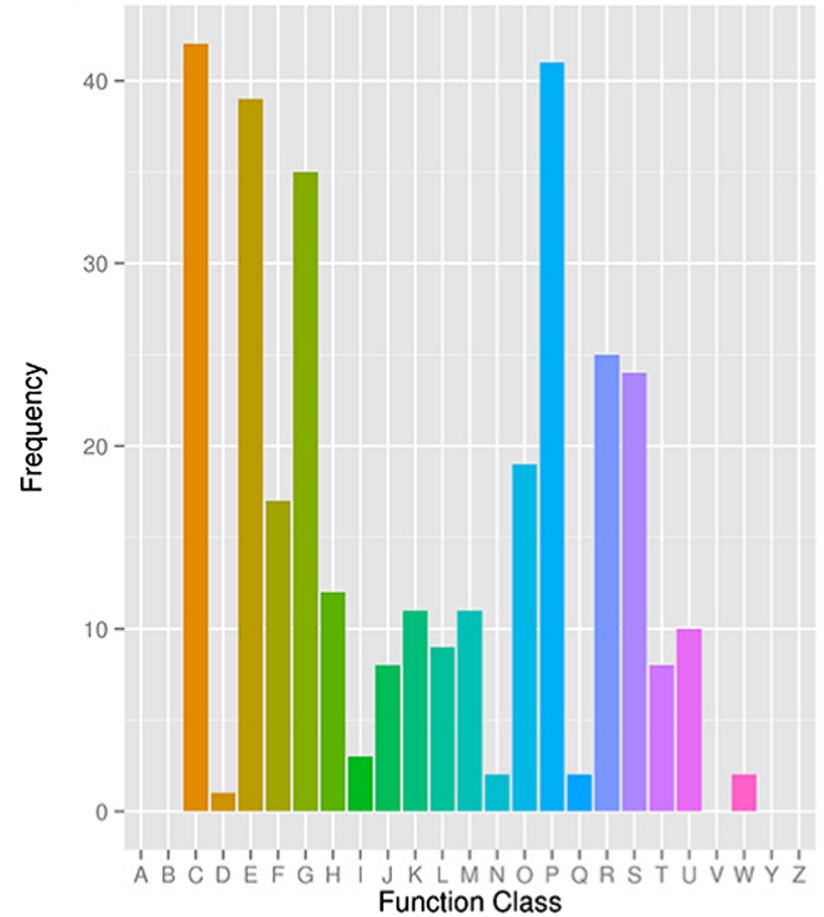

A: RNA processing and modification [0 - 0\%]

B: Chromatin structure and dynamics [0 0\%]

C: Energy production and conversion [42 13.08\%]

D: Cell cycle control, cell division, chromosome partitioning [1 0.31\%]

E: Amino acid transport and metabolism [39 12.15\%]

F: Nucleotide transport and metabolism [17 5.3\%]

G: Carbohydrate transport and metabolism [35 10.9\%]

$\mathrm{H}$ : Coenzyme transport and metabolism [12 3.74\%]

I: Lipid transport and metabolism [3 0.93\%]

$\mathrm{J}$ : Translation, ribosomal structure and biogenesis [8 2.49\%]

K: Transcription [11 3.43\%]

L: Replication, recombination and repair [9 2.8\%]

M: Cell wall/membrane/envelope biogenesis [11 3.43\%]

$\mathrm{N}$ : Cell motility [2 0.62\%]

O: Posttranslational modification, protein turnover, chaperones [19 5.92\%]

$P$ : Inorganic ion transport and metabolism [41 12.77\%]

Q: Secondary metabolites biosynthesis, transport and catabolism [2 0.62\%]

R: General function prediction only [25 7.79\%]

S: Function unknown [24 7.48\%]

T: Signal transduction mechanisms [8 2.49\%]

$\mathrm{U}$ : Intracellular trafficking, secretion, and vesicular transport [10 3.12\%]

V: Defense mechanisms [0 0\%]

W: Extracellular structures [2 0.62\%]

$\mathrm{Y}$ : Nuclear structure [0 0\%]

Z: Cytoskeleton [0 0\%]

Fig. 3 Function classification of the enriched DEGs in the COG database. a DEGs of Mmu. $\mathbf{b}$ DEGs of App

and presentation on the membrane. Furthermore, the MHCI and MHCII pathways in antigen processing and presentation (ko04612) were activated and promoted the recognition of the pathogen by the TCR of CD8 T cells, KIP of NK cells and TCR of CD4 $\mathrm{T}$ cells. 

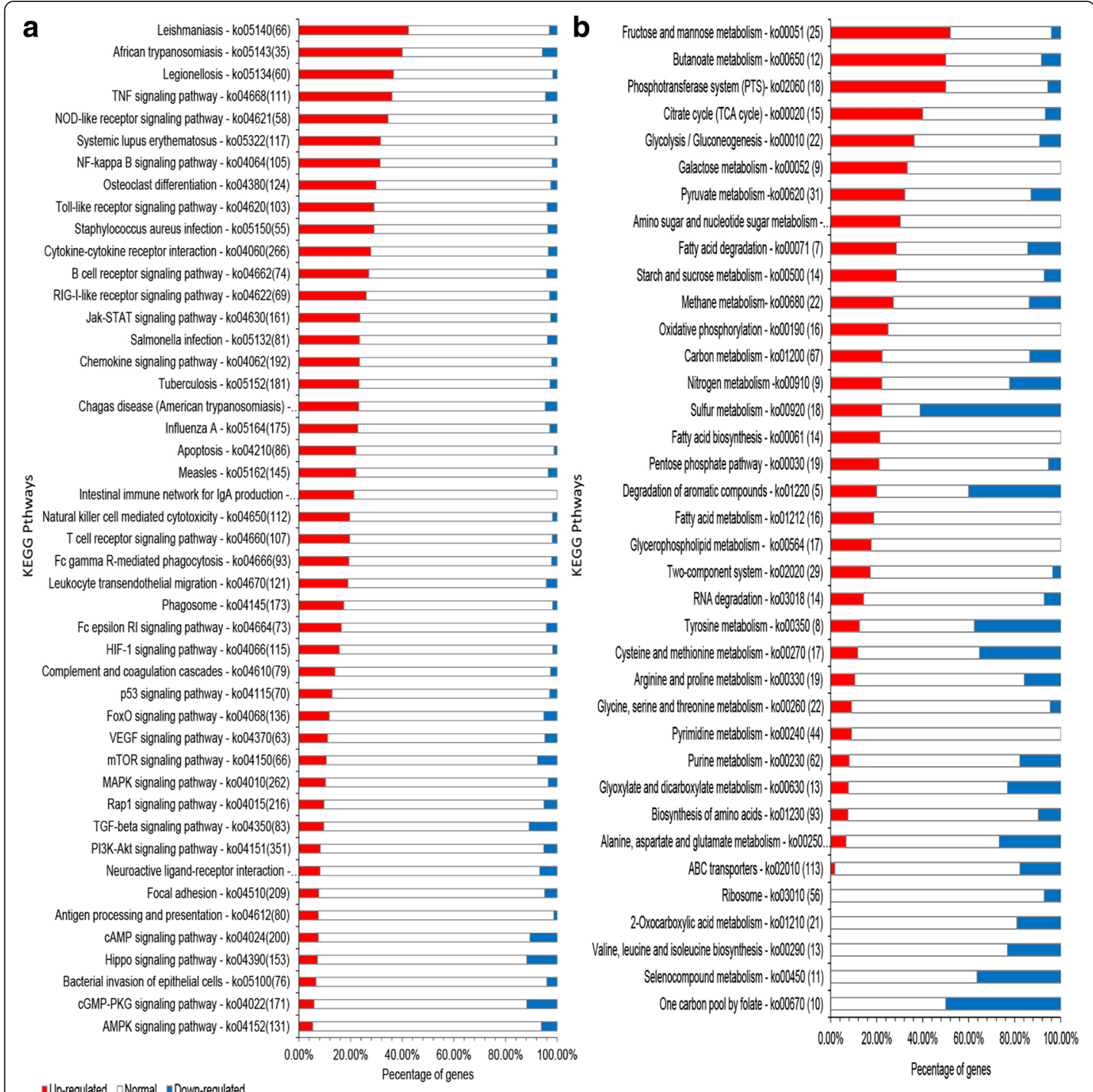

$\backsim$ Up-regulated $\square$ Normal $\quad$ Down-regulated

Fig. 4 Overview of the transcriptional changes in KEGG pathways. a DEGs of Mmu. b DEGs of App

\section{Adaptive immune response: activation of humoral and cellular immunity}

This study found that $27.03 \%$ of genes in the B cell receptor (BCR) signaling pathway (ko04662) were activated, promoting B cell ontogeny, immune response and immunoglobulin production. In addition, in the complement and coagulation cascade pathway (ko04610), the antibodyantigen complex activated genes encoding C2 and C5R1 by cascading effects. Finally, phagocyte recruitment and inflammation were stimulated, and the BCR signaling pathway was further activated. In addition, $19.63 \%$ of the genes in the TCR signaling pathway (ko04660) were upregulated, and genes encoding membrane proteins, including $P D-1, C D 45$ and $C D 36$, were activated, causing the overexpression of Il10, Ifng, Csf 2 and Tnf and promoting the proliferation, differentiation and immune response of T cells.

\section{Intercellular inflammatory signal transduction}

The NF-kB signaling pathway (ko04064) is a key step of these signaling pathways, including the BCR, TCR, IL1R, TNF-R1, RLR, TLR, CD40, RANK and LT- $\beta R$ 


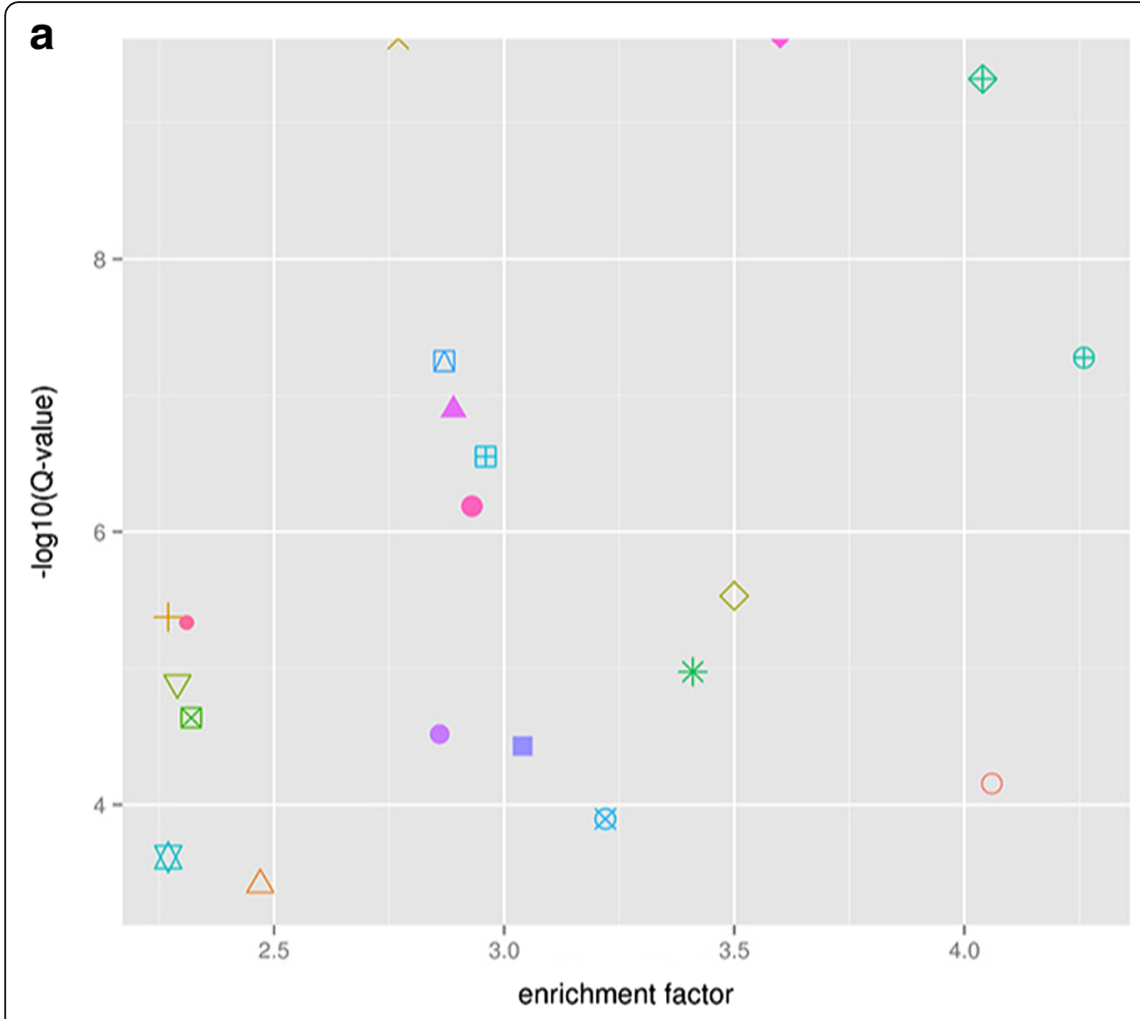

\section{KEGG_pathway}

African trypanosomiasis

$\triangle$ Chagas disease (American trypanosomiasis)

+ Chemokine signaling pathway

$X$ Cytokine-cytokine receptor interaction

$\diamond$ Inflammatory bowel disease (IBD)

$\nabla$ Influenza A

$\triangle$ Jak-STAT signaling pathway

* Legionellosis

$\oplus$ Leishmaniasis

$\oplus$ Malaria

\&8 Measles

$\boxplus$ NF-kappa B signaling pathway

$\nsubseteq$ NOD-like receptor signaling pathway

$\triangle$ Osteoclast differentiation

口 Pertussis

- Rheumatoid arthritis

$\triangle$ Systemic lupus erythematosus

- TNF signaling pathway

Toll-like receptor signaling pathway

- Tuberculosis

b

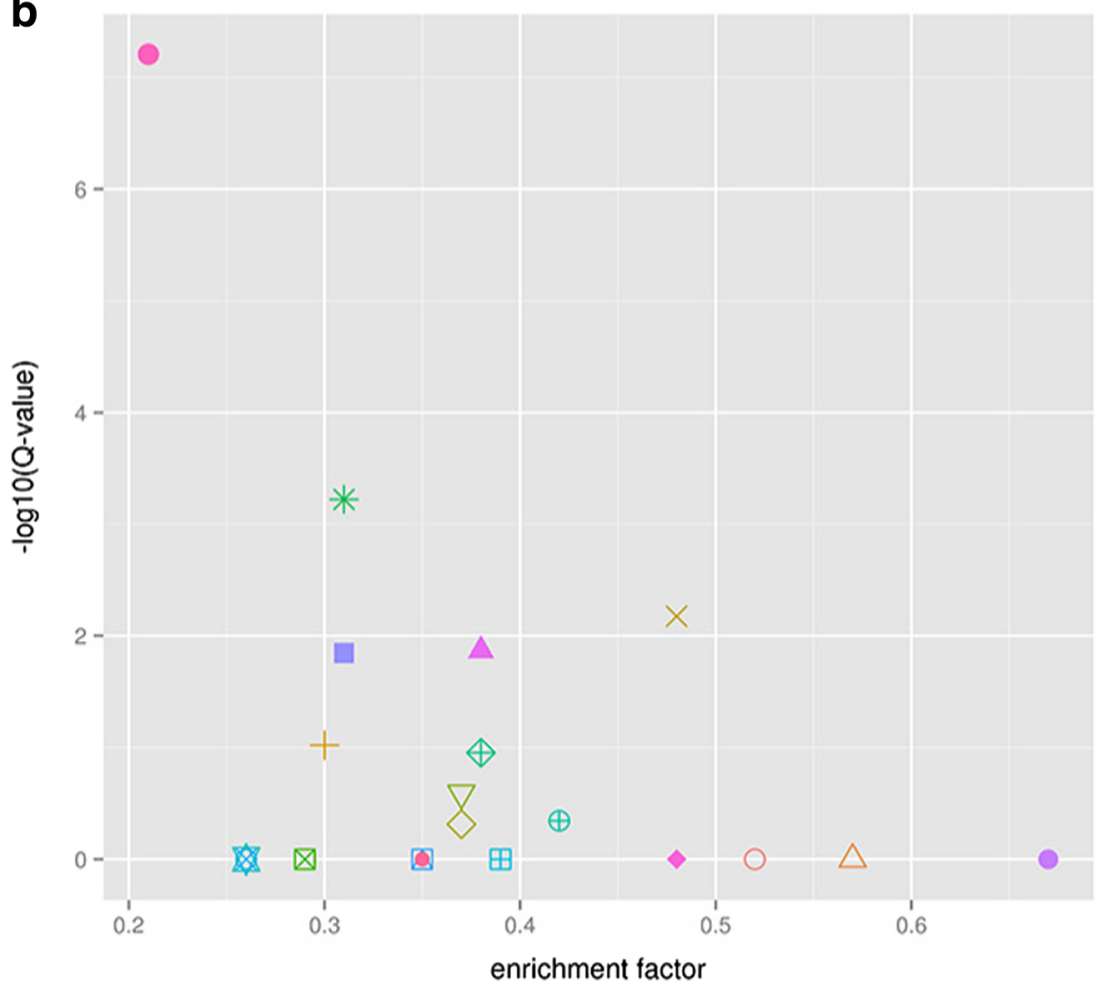

KEGG_pathway

Alanine, aspartate and glutamate metabolism

$\triangle$ Amino sugar and nucleotide sugar metabolism

+ Butanoate metabolism

$X$ Carbon metabolism

$\diamond$ Citrate cycle (TCA cycle)

$\nabla$ Cysteine and methionine metabolism

$\triangle$ Degradation of aromatic compounds

* Fructose and mannose metabolism

$\oplus$ Glycolysis / Gluconeogenesis

$\oplus$ Methane metabolism

\$8 Naphthalene degradation

$\boxplus$ Nitrogen metabolism

$\bowtie$ Nitrotoluene degradation

$\triangle$ One carbon pool by folate

Phosphotransferase system (PTS)

- Purine metabolism

$\triangle$ Pyruvate metabolism

- Starch and sucrose metabolism

- Sulfur metabolism

- Tyrosine metabolism

Fig. 5 Twenty KEGG pathways with a lower enrichment factor and a higher Q value. a KEGG pathways of Mmu. b KEGG pathways of App 


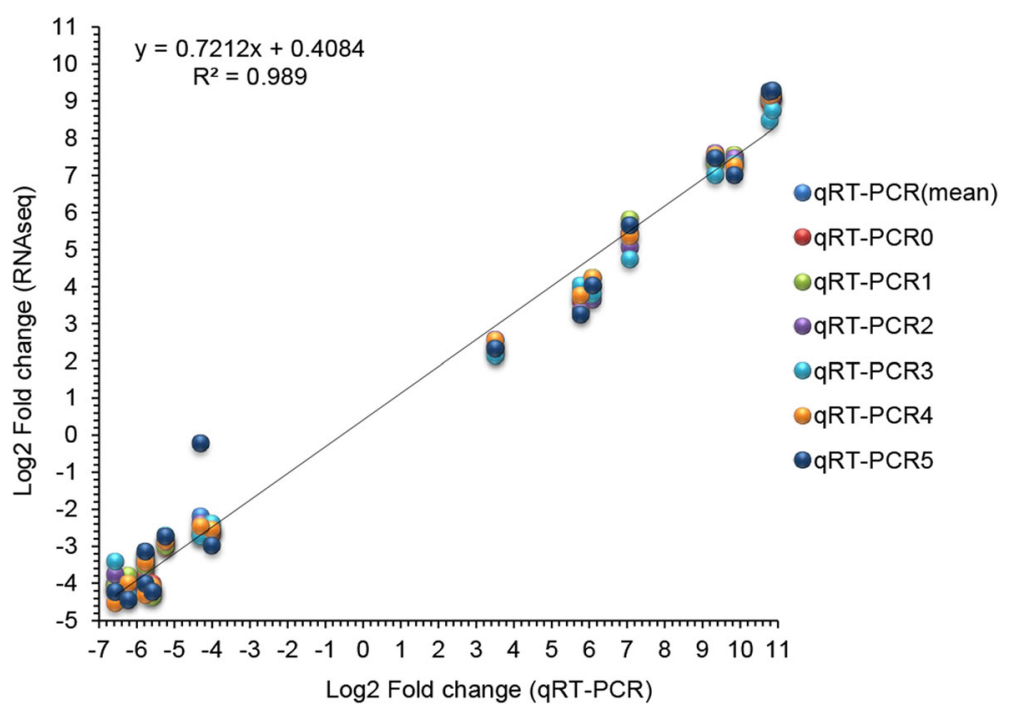

Fig. 6 Comparison of relative expression levels measured by RNA-seq and qRT-PCR. qRT-PCR (mean): the mean of log (fold change) of six RNA samples. qRT-PCR0: the RNA sample used for sequencing. qRT-PCR 1-5: RNA samples mixed in equal parts for sequencing. Of the genes that were found to exhibit significant difference in expression by RNA-seq, the following were selected for detection by qRT-PCR: adhE, dms $A$, dmsB, fruk, malE, lamb, hiuH, metQ1 of App, and cxcl2, csf3, ccl4, ccl3, Tpm3, 60SrDNA, Csnk2a1 and Hba of Mmu. A strong correlation $\left(\mathrm{R}^{2}=0.989\right)$ in gene expression was found between the RNA-seq and qRT-PCR results

pathways, and regulated the transcription of multiple cytokines. We determined that $31.43 \%$ the genes involved in this pathway were up-regulated and caused upregulation of PIP1, c-IAP1/2, c-FLIP, TRAF1/2 and A1/ $B f l-1$ for survival and of COX2, MIP-1 $\beta$ and VCAM-1 for inflammation. In addition, the up-regulated expression of Ifng, IllO, Il2/3 and Il6, which form part of the JAK-STAT signaling pathway (ko04630), activated the expression of Jak and Shp1 by binding to receptors. With respect to the MAPK signaling pathway (ko04010), an important intercellular signal transduction pathway, $10.31 \%$ and $3.44 \%$ of its genes were found to be upregulated and down-regulated, respectively. The JNK and p38 MAP kinase pathway was activated by the bacterial LPS, TNF, IL-1 and FASL TGFB. Some factors in the ERK5 pathway, such as serum, EGF and oxygen species, stimulated host cells and up-regulated Nur77 for proliferation and differentiation.

\section{Pathogenic mechanism of App from bacterial metabolism in vivo}

The annotation of the DEGs in the nr database helped show that most of them were metabolic genes, but their function can only be inferred by referring to other bacteria showing protein homology. In addition, we classified these in the COG database (Additional file 2).

\section{Energy production and conversion mechanisms}

These significantly up-regulated genes were mainly enriched in "energy production and conversion", a term in the COG classification, and were involved in anaerobic respiration, which is similar to the results of some previous studies [15, 22]. In fact, $67.50 \%$ of the genes associated with this term were up-regulated, many are involved in electron transport in respiration (GO:0009055). In addition, the majority of these genes encode hydrogenases and oxidoreductases, such as nitrite reductase $(n r f A B C D)$, periplasmic nitrate reductase (napA), trimethylamine- $\mathrm{N}$-oxide reductase (tor $Y Z)$, dimethylsulfoxide reductase $(d m s A B C D)$, fumarate reductase $(\operatorname{frd} A B C D)$, and nitrate reductase A $(n a r P)$, revealing the respiratory strategies of App, which involve the selection of nitrate, fumarate, dimethylsulfoxide (DMSO) and trimethylamine $\mathrm{N}$-oxide (TMAO) as electron acceptors instead of oxygen. This finding indicates that the bacteria encountered an anaerobic environment during the invasion process. App cultured in isolated necrotic lung tissue or oxygen-deficient culture medium would regulate the expression of enzymes involved in respiratory mechanisms, which play an essential role in metabolic adaptations and virulence [23].

Genes such as adhE, glpAC, napFGH, mah and hybABCDO were highly up-regulated. The up-regulation was particularly obvious for $a d h E$, which was annotated as a bifunctional acetaldehyde-CoA/alcohol dehydrogenase and participates in the biological processes of alcohol metabolism (GO:0006066), carbon utilization (GO:0015976), metal ion binding (GO:0046872) and oxidation-reduction processes (GO:0055114). In addition, proteins encoded by $N a p F, h y b A, g l p C$, nap $G$ and napH have [4Fe-4S] dicluster domains and [4Fe-4S]-binding domains, and $g l p A$ also encodes a protein with a BFD-like [2Fe-2S]-binding domain. These proteins containing $[\mathrm{Fe}-\mathrm{S}]$ diclusters are 
metalloenzymes with abundant biological functions, such as electron transfer and electron delocalization in respiration spin states and magnetism, catalysis in central metabolic pathways, gene regulation in response to the environment and protein structure stabilization [24, 25].

\section{Carbohydrate transport and metabolism}

Carbohydrate metabolism is the major and direct source of energy for organisms. An analysis of carbohydrate transport and metabolism revealed that the proportion of up-regulated genes enriched in this term was $75.00 \%$, and these genes were mainly involved in the metabolism of fructose, mannose, phosphoglycerate, triosephosphate, sucrose, glucose and galactose. Many of these activated enzymes belong to the phosphoenolpyruvate-dependent sugar phosphotransferase system (GO:0009401) and are involved in the biological processes of protein-N(PI)-phosphohistidine-sugar phosphotransferase activity (GO:0008982), integral membrane component (GO:0016021), and ATP binding (GO:0005524), among others.

The most significantly up-regulated genes, namely $p f k A$ and man $X Y Z$, were related to the metabolism of fructose and mannose. The pfkA gene, which in Escherichia coli encodes 6-phosphofructokinase, a key enzyme of glucose metabolism involved in growth under anaerobic conditions, is required in the Embden-Meyerhof pathway [26, 27]. In addition, Escherichia coli overexpressing the $\operatorname{man} X, \operatorname{man} Y$ and man $Z$ genes show higher affinity to various organic solvents, but each these genes does not exert a significant individual effect [28]. These genes also help with glucose utilization, and the manXYZ mutation ( $\operatorname{man} X Y Z$ ) decreases the utilization of glucose but increases that of xylose [29].

\section{Mechanism of inorganic ion transport metabolism}

Many DEGs in the category "inorganic ion transport and metabolism" had commonalities in that they were connected to the transport and metabolism of iron $(29.73 \%)$ and sulfate (35.14\%). Genes associated with this term were mainly down-regulated, with a proportion of $72.97 \%$. These significantly down-regulated genes included TonB-dependent receptors, catalase, formate/nitrite transporter, sulfur transferase and NLPA lipoprotein. Many of these genes are related to the virulence of App, which indicates that these proteins and enzymes are suppressed in the conflict between offense and defense.

\section{Amino acid transport and metabolism}

The DEGs associated with this term were mainly downregulated; in fact, the down-regulation rate was $80.65 \%$. In addition, only five genes, namely $s d a C$, sdaA, $d m s D$, $u r e C$ and $u r e B$, were up-regulated. Both $s d a C$ and $s d a A$ are involved in serine metabolism, which had the most significant differences in amino acid metabolism. It has been reported that serine is preferentially used in amino acid metabolism during growth in complex or defined media by Campylobacter jejuni [30], and this preference is due to three genes $(\operatorname{ser} A B C)$ of the bacteria [31], which are similar in terms of sequence to Escherichia coli sdaA, encoding an L-serine dehydratase. SdaA of $A p p$ also encodes an L-serine dehydratase, with has the biological activity of degrading L-serine into pyruvate and ammonia to provide forms of carbon and nitrogen for the central metabolism. It also has a [4Fe-4S] cluster similar in structure to that of Campylobacter jejuni. The $\mathrm{Fe}^{2+}$ components of these $\mathrm{Fe}-\mathrm{S}$ centers are considered to have the ability to reactivate $\mathrm{L}$-serine dehydratases of some anaerobic bacteria under anaerobic conditions when these are inactivated by exposure to air. In addition, the sdaC protein is a serine transporter and septum formation initiator and belongs to the tryptophan/tyrosine permease family. SdaC in Campylobacter jejuni encodes a low-affinity L-serine-specific transporter and shows a similar structure to those of the Escherichia coli sdaC protein and members of the tryptophan/tyrosine permease family. Based on these data, we conjectured that App could have a preference for serine compared with other amino acids in vivo and that these L-serine dehydratases and serine transporter play vital roles in serine metabolism under anaerobic conditions; however, these inferences require further analyses.

Moreover, this study revealed that $u r e C$ and $u r e B$ were significantly up-regulated. These genes encode urease, which is important for the internal survival functions of the bacteria. Nina Baltes et al. compared the effects of an App mutant ( $\Delta u r e C)$ and its parent strain on the immune system and found that the mutant strain revealed a higher number of App-specific B cells in the BALF, which suggested that the activity of $A p p$ urease might cause inhibition/damage to the immune system of the host to aid in the persistence of the bacteria.

\section{Cell wall/membrane/envelope biogenesis}

Genes involved in bacterial cell wall/membrane/envelope biogenesis were detected, and 10 of these were significantly regulated. Among them, $\operatorname{lrgB}, a \operatorname{lr}, \operatorname{glm} S, \operatorname{murC}$ and murG were up-regulated. The up-regulated genes alr, murC and murG, which encode the components of amino acid ligases, racemases and GTases, respectively, are involved in the synthesis of the peptidoglycan precursor [32]. Similarly, glmS encodes glucosaminefructose-6-phosphate aminotransferase, and one of its catalytic products, $\mathrm{N}$-acetylglucosamine, is an essential constituent of the bacterial peptidoglycan layer [33]. $\operatorname{Irg} B$ encodes a murein hydrolase regulator, and the protein also could regulate the growth of the bacterial cell wall. 
These murein hydrolases play a major role in the course of infection by releasing highly bioactive cell wall subcomponent autolysins [34].

\section{Identification of hypothetical proteins}

Many DEGs had no available biological information or annotation details. All of the identified DEGs encoding hypothetical proteins, with the exception of APP7_RS05355, were down-regulated. A novel gene (novel 4) could be annotated in $\mathrm{nr}$ as an unknown protein of Mannheimia succiniciproducens.

\section{Discussion}

In this study, a mouse model was established for the study of App infection. In addition, the results showed many similarities between mouse and pig in terms of clinical symptom, pathological changes of the lungs and the immune response, such as the activation of signaling pathways and the regulation of some cytokines. These findings show the applicability of the mouse model of App infection and provide a basis for further analysis. Our study presents a global view of the transcriptional profiling of App in the mouse host-lung model and further reveals the interactions between the bacteria and the host based on a large dataset of transcriptional information obtained by RNA-seq.

In addition, to capture many more transcripts of App in vivo from the complex transcriptional information arising from host interference, we selected to enrich the bacteria in the lung tissue through intranasal inoculation. We selected the infected lungs with the highest content of App, as detected by RT-qRCR, for sequencing, and increased the sequencing volume to ensure the accuracy and integrity of the results. We ultimately gained $15 \mathrm{~Gb}$ of clean data. These clean data were compared with the reference genome of App serotype 7, showing a comparison efficiency up to $15.68 \%$, which is conducive for the subsequent analysis. As a result, we identified many significant DEGs that had never been previously reported. This study provides a basis and reference for further analysis of the physiological activity of App in vivo, particularly for the foundation of pathogenesis and immune evasion in App. The study also analyzed the transcriptional information of host lung tissue to reveal the anti-infection mechanism of the host.

\section{Activation of an anti-infection cytokine cascade}

In the current study, the bacterial pathogens activated PRR signaling pathways, such as the TLR, NLR, and RLR signaling pathways. These bacterial pathogens include bacterial peptidoglycan, bacterial RNA, poreforming toxin, flagellin, lipoarabinomannan, lipoprotein, lipopolysaccharide, and imidazoquinoline. The activation of these signaling pathways promoted the up-regulation of genes encoding cytokines, including granulocyte colony-stimulating factor, $\mathrm{C}-\mathrm{X}-\mathrm{C}$ motif chemokine, interleukin, C-C motif chemokine, tumor necrosis factor (TNF) and interferon gamma (IFN $\gamma$ ), which are listed in Additional file 3, and the fold-change in expression ranged 5 to 1871 . The up-regulation of these cytokines on such a large-scale demonstrates the cytokine cascade involved in the host immune system.

Some of these cytokines are pro-inflammatory factors, such as IL-6, IL-1 and TNF, and their up-regulated expression increases the inflammatory response. In particular, the up-regulated expression of IL- $1 \beta$ and TNF activates the $\mathrm{NF}-\mathrm{\kappa} \mathrm{B}$ signaling pathway and provides positive feedback to this pathway. Similarly, TNF activates the TNF signaling pathway and releases many cytokines, including those associated with leukocyte recruitment and activation factors, as well as inflammatory cytokines and transcription factors. The up-regulation of TNF also provides positive feedback to the pathway. Tnf and $I l 1 b$, which were found to be upregulated in this study, could promote the transcription and expression of Ptx3, a humoral pattern recognition receptor and nonredundant component of humoral innate immunity for anti-infection [35]. It has been reported that IL-10, which is known for its anti-inflammatory activity, could also stimulate the secretion of PTX3 protein, presenting pro-inflammatory activity, whereas IFN- $\gamma$ could inhibit the production of PTX3 [36]. Similarly, the acknowledged pro-inflammatory cytokines IL-6 and TNF $\alpha$ have also been reported to have anti-inflammatory abilities in the defense against Mycobacterium tuberculosis infection [37, 38]. The interactions of these cytokines are complex, and the role of anti-infection could be different in other bacteria, but the positive feedback of the signaling pathways could promote the observed cytokine cascade.

In addition, the IL-23/IL-17 axis was found to play a significant role in the host defense against bacterial infection in the present study. Cytokines such as IL-23, IL-17A, IL-17F, IL-1, IL-6, Csf2 and CXC are crucially regulated points of the IL-23/IL-17 axis and the genes encoding them were found to be highly upregulated. IL-23 could regulate innate and adaptive immune responses and is produced by dendritic cells and macrophages rapidly when these are stimulated by bacterial pathogens. IL- 23 and IL- $1 \beta$ activate Th17/Th $\mathrm{IL-17}$ and other IL-17-producing cells, such as natural killer $\mathrm{T}$ (NKT) cells, which could recognize microbial glycosylceramides, a component of the cell wall of Gram-negative bacteria, to release IL-17, which could induce the secretion of G-CSF [39, 40]. As a result, the IL-23/IL-17/G-CSF pathway is activated to augment neutrophils for bacterial removal [41]. IL-23 also promotes the expression of proinflammatory cytokines, such as IL-1, IL-6 and TNF [42], and some inflammatory factors, such as Csf2 
(GM-CSF), Ccl20, Ccl22, IL-1 and IL-23. Th17 cells, a key player in autoimmune diseases and antibiosis, are differentiated from naïve $\mathrm{T}$ cells $(\mathrm{CD} 4+)$ stimulated by IL-6, TGF- $\beta$, IL-1 $\beta$, IL-21 and IL-23 [43-46] and release IL-17A, IL-17F, IL-21 and IL-22. Together with TGF- $\beta$, IL- 6 can induce the generation and maturity of Th17 cells and can inhibit TGF- $\beta$-mediated regulatory $\mathrm{T}$ cell (Treg) differentiation to regulate the Th17/Treg balance [47]. IL-22 has been reported to have an essential ability in recruiting neutrophils and soluble antibacterial factors to aid the response against bacterial infection [48], such as infection by N. gonorrhoeae [49] and Citrobacter rodentium [50]. Both IL-22 and IL-17 (IL-17R signaling) have been demonstrated to play crucial roles in maintaining the local control of Gram-negative pulmonary pathogens and regulate the secretion of CXC chemokines [51, 52], which are involved in the recruitment of neutrophils against bacterial pneumonia. Previous studies have confirmed that the IL-23/IL-17 axis of the host exerts antibacterial effects, and the present study demonstrates its involvement in the response to infection by App.

\section{Cytokine cascade could cause lung damage during infection}

The infection with App caused acute bacterial pneumonia with infiltration by immune cells, including macrophages, neutrophils, eosinophils and lymphocytes, and released a large amount of inflammatory mediators through a cytokine cascade to defend against the infection. However, it also caused damage to pulmonary cells and tissues directly or indirectly. For instance, TNF $\alpha$, which has the function of mediating components of the acute-phase response and stimulating granulocyte metabolism, has been proven to cause acute pulmonary vascular endothelial injury [53]. IL-6 is usually highly expressed when cells are stimulated by bacterial LPS, viruses and cytokines such as IL-1, IFN and TNF $\alpha$, and the concentration of IL- 6 and TNF $\alpha$ can reflect the severity of pneumonia $[54,55]$. In the present study, the histopathological symptoms and high expression of Il6, Ill, Ifng and Tnf demonstrated that the host was suffering from serious acute bacterial pneumonia, which could be caused by the infection and the detected cytokine cascade.

\section{Anaerobic metabolism of App was activated in serious acute bacterial pneumonia}

Serious bacterial pneumonia could lead to the disturbance of microcirculation and tissue blood perfusion, resulting in the failure of microcirculation and the onset of histanoxia. Many of the DEGs identified in this study are involved in anaerobic metabolism, which indicates that App in the infected lung was under anaerobic stress. Previous studies have demonstrated that genes such as $d m s A, \operatorname{asp} A$ and $h l y X$ are essential for the virulence, inner survival and persistent existence of $A p p$ in vivo by encoding dimethyl sulfoxide (DMSO) reductase [56], the aspartate ammonia lyase [57] and the transcriptional regulator FNR [58, 59], respectively. Many genes or enzymes involved in oxidative metabolism under anaerobic conditions are virulence genes or help regulate the expression of virulence genes. This study detected some genes that were significantly up-regulated but lacked profiles. Their details were few in App but numerous in other bacteria. For instance, adhE of Escherichia coli is essential for ethanol fermentation [60] and affects the virulence of bacteria under anaerobic respiration. By constructing an Escherichia coli mutant ( $\triangle a d h E)$, researchers found that $a d h E$ affects the expression of virulence genes, reduces the ability to adhere to host cells and activates identification of TLR-5 [61, 62]. This gene is also expressed in an aerobic environment [63], but its role is undefined. Genes such as $\operatorname{glpA}$, NapF, hybA, glpC, napG, napH, pfkA, CitT, and sdaA, have been reported as important proteins in anaerobic metabolism, but their connection to bacterial virulence needs further research.

\section{In vivo immune evasion strategies of $A p p$}

The bacterial cell wall and envelope constitute the first line of defense against attacks by the host immune system. In addition, the up-regulation of alr, murC, murG and $g \operatorname{lm} S$ identified in this study promotes the biogenesis of peptidoglycan and helps maintain the cell shape [64]. Boneca et al. demonstrated that peptidoglycan modifications, including amidation, acetylation and glycosylation [65], could help Listeria gain resistance in the host by immune evasion [66], but this requires further study in App. In addition, genes encoding urease, which helps hydrolyze urea to ammonia and water, were found to be up-regulated, and ammonia could enhance neutrophil-dependent cell damage and cause inactivation of $\mathrm{C} 4$ of the complement cascade for immune evasion $[67,68]$.

Notably, the major virulence genes of App identified in the present study were down-regulated or showed no significant difference in expression. These include genes that encode RTX toxins, capsule proteins, outer membrane proteins and transferrin-binding proteins. Most of these proteins are present on the membrane and could be recognized by the host and eliminated. This result is similar to those obtained by Deslandes et al., who found that important virulence genes, including $c p x A B C D$, apxI $B C D$, apxII $A B$ and Tbp $A B$, showed no significant 
difference in expression. By constructing gene deletion strains, these researchers found that the absence of capsule genes contribute to the adhesion of App in host cells [69]. The restricted expression of these genes might be a strategy of App for in vivo immune evasion.

\section{Hypothesis of the dominant antigens expressed in different phases}

Based on the differential expression of App genes identified in our and other studies, we inferred that different and specific dominant antigens are expressed at different phases of App infection, and these could thus be considered vaccine candidates instead of or in addition to major virulence genes. In the early phase of infection, bacteria are adapting to the host environment, and a precise and targeted immune response would be advantageous to the rapid removal of the pathogen. Therefore, more studies on the transcriptional profiling of different infection phases should be performed to detect vaccine candidates.

\section{Methods}

\section{Animal ethics statement}

All animal procedures used in the present study were conducted in accordance with good animal practices as defined by the laboratory animal use license (Certificate No. SYXK (CHUAN) 2014-187). All work with mice was approved and supervised by the Committee on the Care and Use of Laboratory Animals of Sichuan and was conducted in the Animal Biotechnology Center of Sichuan Province at Sichuan Agricultural University. The animal interventions were performed in strict accordance with animal ethical standards. In addition, to limit any suffering, all mice used for lung collection were euthanized by rapid decapitation at the endpoint, which could also reduce the hemocytes in the tissues used for histopathological analysis.

\section{Animals and bacterial strains}

The SPF KM mice (Mus musculus, provided by Chengdu Dashuo Biological Technology Co. Ltd.) used in the study were similar in age and closely related. They weighed 25-28 g and were housed in appropriate containment facilities with feed and water provided ad libitum.

App serotype 7 (CVCC265, preserved in China Institute of Veterinary Drug Control) isolated from pigs with acute pleuropneumonia was used for the infection study. The strain was cultured at $37{ }^{\circ} \mathrm{C}$ on tryptone soy broth (TSB) with $15 \mu \mathrm{g} / \mathrm{ml} \mathrm{NAD}$ and $10 \%$ calf serum. When the bacterial strain reached the exponential phase in $\mathrm{TSB}$, it was harvested at $4{ }^{\circ} \mathrm{C}$ by low-speed centrifugation $(4000 \mathrm{rpm} \times 15 \mathrm{~min})$. A portion of the bacterial cells were collected and rapidly frozen in liquid nitrogen for RNA-seq, and the rest of the cells were suspended with PBS to a final concentration of $2.69 \times 10^{10}$ cfu viable bacteria/ml and used for infection.

\section{Infection studies}

In the study, 35 SPF $M m u$ were infected with App serotype 7 by intranasal inoculation at a total dose of $5.38 \times 10^{9} \mathrm{cfu}\left(0.2 \mathrm{ml}, 10 \times \mathrm{LD}_{50}\right)$, and these mice constituted the treatment group. Twenty-five SPF $M m u$ served as the control group and were treated with PBS in the same manner. After infection, the two groups were housed separately in appropriate containment facilities in the laboratory, with feed and water provided ad libitum. In addition, their intake of food and water and their mental status were recorded once per hour.

The infection made the animals weaker, and the dyspnea worsened over time. To ensure that the infection successfully caused acute hemorrhagic pneumonia, their lungs were collected for histopathological observation. In addition, to limit their suffering and observed the typical pathological symptoms, the infected animals were euthanized by rapid decapitation at different time points until $12 \mathrm{~h}$ after infection and before their natural death. Seven animals from the treatment group were sacrificed $4 \mathrm{~h}, 6 \mathrm{~h}, 8 \mathrm{~h}, 10 \mathrm{~h}$ and $12 \mathrm{~h}$ post-infection, respectively, and their whole lungs were isolated. In addition, five animals from the control group were sacrificed in the same manner at the same time points.

From each whole lung, a section was rapidly frozen in liquid nitrogen for RNA isolation to estimate the content of the bacterial transcriptome, and the rest was fixed in $4 \%$ paraformaldehyde fixative solution to prepare paraffin sections and stained with HE for histopathological analysis.

\section{RNA isolation and reverse transcription}

The total RNA from the infected lungs was extracted using an RNAiso Plus chloroform-isopropanol-ethanol extraction protocol. The RNA purity, concentration and integrity were measured using a Nano Drop spectrophotometer (Thermo Scientific), a Qubit 2.0 instrument (Invitrogen) and an Agilent 2100 Bioanalyzer (Agilent Technologies), respectively. The quality requirement was $2.0 \geq$ A260/A280 $\geq 1.8$ and RIN (RNA integrity number) $>5$. Otherwise, a new extraction was performed. The resulting high-quality RNA was used for reverse transcription. All of the RNA samples from the lungs were diluted to $100 \mathrm{ng} / \mu \mathrm{l}$ with $\mathrm{dd}_{2} \mathrm{O}$, and $10 \mu \mathrm{g}$ of each sample was used for reverse transcription with an RNA Library Prep Kit for Illumina (NEBNext ${ }^{\circ}$ Ultra $^{\mathrm{m}}{ }^{\mathrm{m}}$ ). A qRT-PCR method ( $\mathrm{y}=-3.313 \mathrm{X}+40.228)$ based on the $16 S r D N A$ of App was performed to calculate the content of App in the RNA samples, and the samples with the five highest contents were selected for further RNA sequencing. 


\section{Library preparation and RNA sequencing}

The high-quality RNA samples were reverse transcribed and used for library construction. The prokaryotic rRNA was removed by probes showing complementarity to rRNA, and the rRNA-depleted RNA could then be enriched. The eukaryotic mRNA was directly enriched by magnetic beads with Oligo(dT) from the total RNA. The details of the library preparation are described in the instructions provided by the RNA Library Prep Kit for Illumina (NEBNext ${ }^{\circ}$ Ultra $^{\mathrm{Tw}}$ ). For RNA sequencing, we used Illumina HiSeq2500, a high-throughput sequencing platform that can provide high-quality raw data (reads) with a base calling accuracy greater than $99.9 \%$. Removal of the sequencing joints and primer sequences and filtering out low-quality data helped us achieve high-quality clean reads.

\section{Analysis of read mapping and gene expression}

The clean reads were aligned against their reference genome by Bowtie and TopHat2 $[70,71]$. The reference genomes of App and $M m u$ could be downloaded from the NCBI database with the ID numbers NC_010939.1 and GCA_000001635.7, respectively. The successfully aligned reads were considered mapped reads, and the rate of mapping (mapped reads divided by total reads) was used to evaluate the data reliability of the selected reference genome assembly. RNA sequencing showed high sensitivity in the detection of gene expression, and the level of transcription or gene expression was measured by RPKM, the coverage of which ranged from $10^{-2}$ to $10^{4}[72,73]$.

\section{Screening and functional analysis of DEGs}

DEGs obtained from the analysis of host genes in infected and healthy lungs (control) and App genes in in vivo and in vitro cultures (control) were defined using EBSeq software [74]. The Benjamini-Hochberg method was used for correcting multiple comparisons, and the DEGs were defined based on fold change $\geq 2$ and FDR $<0.01$. The functional annotation of DEGs was performed based on various databases, including the GO, COG, KEGG, Swiss-Prot, $\mathrm{nr}$ and Pfam databases.

\section{Comparison of RNA-seq and qRT-PCR results}

The RNA sequencing results for transcript profiling were verified by qRT-PCR using SYBR $^{\circ}$ Premix Ex $\mathrm{Taq}^{\mathrm{TM}}$ II $(2 \times)$ (TaKaRa) and the same RNA samples that were used for RNA sequencing. The qRT-PCRs were conducted on a BIO-RAD CFX, and each reaction was performed in a total volume of $25 \mu \mathrm{l}$ containing $12.5 \mu \mathrm{l}$ of $\mathrm{SYBR}^{\circ}$ Premix Ex Taq ${ }^{\text {ti }}$ II $(2 \times)$, $2 \mu \mathrm{l}$ of the cDNA template, $1 \mu \mathrm{l}$ of each primer $(0.5-$ $2.5 \mu \mathrm{l}$ ), and $8.5 \mu \mathrm{l}$ of $\mathrm{ddH}_{2} \mathrm{O}$. All of the reactions were performed in triplicate, and the reaction system was optimized by adjusting the concentrations of the primers and template.

The primers for qRT-PCRs were designed by Primer Premier 5.0 and Primer-BLAST (Additional file 4). The thermal cycling conditions were as follows: $95{ }^{\circ} \mathrm{C}$ for $20 \mathrm{~s}, 40$ cycles of $95{ }^{\circ} \mathrm{C}$ for $10 \mathrm{~s}, 55^{\circ} \mathrm{C}$ for $30 \mathrm{~s}$, and $72{ }^{\circ} \mathrm{C}$ for $30 \mathrm{~s}$, with fluorescence detection at the end of each cycle, and a melting curve analysis (from $60{ }^{\circ} \mathrm{C}$ to $95{ }^{\circ} \mathrm{C}$, increasing at a rate of $1{ }^{\circ} \mathrm{C} / 3$ s) to ensure that each reaction amplified a single and specific product. The qRT-PCRs showed high amplification efficiency (between 95\% and 105\%). Two reference genes, the bacterial $16 \mathrm{~S}$ ribosomal and GADPH genes of $\mathrm{Mmu}$, which showed only a slight variation in the RNA-seq analysis, were used for data normalization.

\section{Conclusions}

To obtain insights into the interactions between App and its host and provide a profile of the pathogenesis and adaptive metabolism of App and the immune and anti-infection responses of the host, an infection model of acute bacterial pneumonia in SPF $М m u$ was established. The infected lungs were isolated for dual RNA-seq, and the transcriptional differences of $A p p$ and $M m u$ between infection and non-infection conditions were revealed. In total, $2428 \mathrm{Mmu}$ genes and $333 \mathrm{App}$ genes were identified as DEGs. The identified host DEGs were mainly up-regulated and activated many immune signaling pathways, such as the TLR, NLR, RLR, RIG-I, BCR, and TCR signaling pathways, and caused upregulation of $C s f 2, C x c l 2$, Il6, Ccl4, Illb, Il23 $\alpha, I l 1 \alpha, I l 10$, Ill7f, Il17a, Tnf, and Ifng, among other cytokines, leading to an anti-infection cytokine cascade and causing lung damage. Most of these cytokines are involved in the IL23/IL-17 cytokine interaction network, which might play a significant role for the host in $A p p$ infection. The DEGs of App were mainly involved in anaerobic metabolism. These include adhE, a metabolic gene that has been proven to be virulence gene in Escherichia coli, $s d a C$, dms $A, d m s B$, tor $Y$, tor $Z$, and $g l p A$. Furthermore, $A p p$ was found to exhibit various strategies for immune evasion, such as enhancing the cell wall and damaging immune cells by synthesizing urease. The strategies regarding the restriction of major virulence genes provide novel vaccine candidates; specifically, different infection phases might be characterized by different dominant antigens, and these dominant antigens might aid the establishment of a precise and targeted immune response during the early phase of infection. These findings are meaningful for the study of App infection, but the differences between mice and pig exist indeed, so more verifications to present their roles in pigs need to be performed in the further study. 


\section{Additional files}

Additional file 1: Activated signaling pathways involved in the inflammatory response. These inflammatory signaling pathways were regulated after infection and directly present based on the regulation of their genes, including up-regulation and down-regulation. (PDF 168 kb)

Additional file 2: DEGs of App classified by COG terms and annotated in the $\mathrm{nr}$ database. These DEGs of App were mainly metabolic genes and annotated in the nr database. They were classified into COG terms to clearly analyze their functions. (PDF $200 \mathrm{~kb}$ )

Additional file 3: Differentially expressed cytokines of the host. These DEGs of the host could be annotated in the nr database as cytokines, and the $\log _{2}$ (fold change) values were used to present their expression level. (PDF $170 \mathrm{~kb}$ )

Additional file 4: Gene-specific primers for qRT-PCR. Significant DEGs of App and Mmu were selected to test the RNA-seq results. The DEGs of App included adhE, dmsA, dmsB, fruK, malE, lamB, hiuH, MetQ1, and the DEGs of Mmu included Cxc12, Csf3, Ccl4, Ccl3, Tpm3, 60SrDNA, Csnk2a1, Hba. 16SrDNA and GAPDH were used as the reference genes of App and Mmu, respectively. (PDF $182 \mathrm{~kb}$ )

\section{Abbreviations}

App: Actinobacillus pleuropneumonia; BCR: B cell receptor; COG: Clusters of Orthologous Groups; CTL: Cytotoxic lymphocyte; DEGs: Differentially expressed genes; FC: Fold change; FDR: False discovery rate; FPKM: Fragments per kilobase of exon model per million mapped reads; GO: Gene Ontology; IL: Interleukin; KEGG: Kyoto Encyclopedia of Genes and Genomes; Mmu: Mus musculus; NK: Natural killer cell; NLR: NOD-like receptor; PAMPs: Pathogen-associated molecular patterns; PRRs: Pattern recognition receptors; RLR: RIG-I-like receptor; SPF: Specific pathogen-free; TCR: T cell receptor; TLR: Toll-like receptors

\section{Acknowledgements}

We thank Prof. Zhu for the insightful comments regarding the design of the study and the manuscript.

\section{Funding}

This present study was supported by the Program for Changjiang Scholars and Innovative Research Team University (IRT13083).

\section{Availability of data and materials}

The data supporting the conclusions discussed in this article are included within the article and its additional files. The datasets used and/or analyzed in the current study are available in NCBI (SRR5936634, SRR5936635 and SRR5931780).

\section{Authors' contributions}

$\mathrm{PL}, \mathrm{ZWX}$ and $\mathrm{LZ}$ conceived the study, designed the experiments and reviewed the manuscript. $P L$ performed the experiments and drafted the manuscript. PL, XGS, YY, YF, and JZ analyzed the data. XYM, JBH, and FY collected software and prepared the figures and tables. The final manuscript has been read and approved by all of the authors.

\section{Ethics approval}

All animal procedures used in the present study were conducted in accordance with good animal practices as defined by the laboratory animal use license (Certificate No. SYXK (CHUAN) 2014-187). All work with mice was approved and supervised by the Committee on the Care and Use of Laboratory Animals of Sichuan and was conducted in the Animal Biotechnology Center of Sichuan Province at Sichuan Agricultural University.

\section{Consent for publication}

Not applicable.

\section{Competing interests}

The authors declare that they have no competing interest.

\section{Publisher's Note}

Springer Nature remains neutral with regard to jurisdictional claims in published maps and institutional affiliations.

Received: 31 May 2017 Accepted: 5 September 2017

Published online: 12 September 2017

\section{References}

1. Bossé JT, Janson H, Sheehan BJ, Beddek AJ, Rycroft AN, Kroll JS, et al. Actinobacillus pleuropneumoniae: pathobiology and pathogenesis of infection. Microbes Infect. 2002;4(2):225-35.

2. Halli $O$, Ala-Kurikka E, Wallgren $P$, Heinonen M. Actinobacillus pleuropneumoniae seroprevalence in farmed wild boars in Finland. J Zoo Wildl Med. 2014;45(4):813-8.

3. Cai-Xia FU, Jin XJ, Yuan L, Jia LI, Chun-Hua YU. Serological investigation on porcine contagious Pleuropneumoniae in some areas of Beijing. Progress Vet Med. 2009;30(5):109-11.

4. Shabunin SV, Stepanov AV, Efanova LI, Manzhurina OA. Serological profile of Actinobacillus pleuropneumoniae in pigs. Russ Agric Sci. 2012;38(38):406-8.

5. Bröring S, Müller E, Petzoldt K, Schoon HA, Bergmann KC. The synergism of Actinobacillus pleuropneumoniae and influenza a virus in experimentallyinfected mice. Zentralb| Veterinarmed B. 1989:36(9):681-90.

6. Dayao D, Gibson J, Blackall P, Turni C. Antimicrobial resistance genes in Actinobacillus pleuropneumoniae, Haemophilus parasuis and Pasteurella multocida isolated from Australian pigs. Aust Vet J. 2016;94(7):227-31.

7. Vanni M, Merenda M, Barigazzi G, Garbarino C, Luppi A, Tognetti R, et al. Antimicrobial resistance of Actinobacillus pleuropneumoniae isolated from swine. Vet Microbiol. 2012;156(1-2):172

8. Nedbalcova K, Satran P, Jaglic Z, Ondriasova Z. Kucerova, et al. monitoring of antibiotic resistance in isolates of Actinobacillus pleuropneumoniae in the Czech Republic between 2001 and 2003[J]. Vet Med Czech. 2005;50:181-5.

9. Shakarji L, Mikael LG, Srikumar R, Kobisch M, Coulton JW, Jacques M. Fhua and $\mathrm{HgbA}$, outer membrane proteins of Actinobacillus pleuropneumoniae: their role as virulence determinants. Can J Microbiol. 2006;52(4):391-6.

10. Baltes N, Tonpitak W, Gerlach G, Hennigpauka I, Hoffmannmoujahid A, Ganter M, et al. Actinobacillus pleuropneumoniae iron transport and urease activity: effects on bacterial virulence and host immune response. Infect Immun. 2001;69(1):472-8.

11. Xu F, Chen X, Shi A, Yang B, Wang J, Li Y, et al. Characterization and immunogenicity of an apxIA mutant of Actinobacillus pleuropneumoniae. Vet Microbiol. 2006;118(3-4):230-9.

12. Ramjeet M, Deslandes V, Goure J, Jacques M. Actinobacillus pleuropneumoniae vaccines: from bacterins to new insights into vaccination strategies. Anim Health Res Rev. 2008:9(1):25-45.

13. Schaller A, Kuhn R, Kuhnert P, Nicolet J, Anderson TJ, Macinnes Jl, et al. Characterization of apxIVA, a new RTX determinant of Actinobacillus pleuropneumoniae. Microbiology. 1999;145(Pt 8):2105-16.

14. Deslandes V, Denicourt M, Girard C, Harel J, Nash JHE, Jacques M Transcriptional profiling of Actinobacillus pleuropneumoniae during the acute phase of a natural infection in pigs. BMC Genomics. 2010;11(1):98.

15. Klitgaard K, Friis C, Jensen TK, Angen $\varnothing$, Boye M. Transcriptional portrait of Actinobacillus pleuropneumoniae during acute disease-potential strategies for survival and persistence in the host. PLoS One. 2012;7(4):e37040.

16. Zuo Z, Cui H, Li M, Xi P, Ling Z, Ming Z, et al. Transcriptional profiling of swine lung tissue after experimental infection withActinobacillus pleuropneumoniae. Int J Mol Sci. 2013;14(5):10626-60.

17. Brogaard L, Klitgaard K, Heegaard PMH, Hansen MS, Jensen TK, Skovgaard K Concurrent host-pathogen gene expression in the lungs of pigs challenged with Actinobacillus pleuropneumoniae. BMC Genomics. 2015:16(1):417.

18. Teixeira PJ, Thomazella DP, Reis O. Do Prado PF, do Rio MC, Fiorin GL, et al. high-resolution transcript profiling of the atypical biotrophic interaction between Theobroma Cacao and the fungal pathogen Moniliophthora perniciosa. Plant Cell. 2014:26(11):4245-69.

19. Wang Z, Gerstein M, Snyder M. RNA-Seq: a revolutionary tool for transcriptomics. Nat Rev Genet. 2009;10(1):57-63.

20. Zhang J, Xing S, Chang C, Qi X, Chang J, Bi Y, et al. Establishment of to Actinobacillus Pleuropneumoniae of mice model on porcine contagious Pleuropneumonia. J Heilongiiang Bayi Agric Univ. 2015;27(6):19-23.

21. Liu YX, Hai-Bing HU, Shu-Qing LI, Cui L. Establish Actinobacillus pleuropneumonise type 1 infection model in mice and Odserve Pathologicdl changes. J Shanghai Jiaotong Univ. 2015;33(5):1-5. 
22. Bongaerts J, Aachen F. Alternative respiratory pathways of Escherichia Coli: energetic and transcriptional regulation in response to electron acceptors. Biochim Biophys Acta. 1997;1320(3):217-34.

23. Price CE, Driessen AJ. Biogenesis of membrane bound respiratory complexes in Escherichia Coli. Biochim Biophys Acta. 2010;1803(6):748-66.

24. Beinert H. Iron-sulfur proteins: ancient structures, still full of surprises. J Biol Inorg Chem. 2000;5(1):2-15.

25. Johnson DC, Dean DR, Smith AD, Johnson MK. Structure, function, and formation of biological iron-sulfur clusters. Biochemistry. 2005;74(74):247-81.

26. Steinbüchel A. Expression of the Escherichia Coli pfkA gene in Alcaligenes Eutrophus and in other gram-negative bacteria. J Bacteriol. 1986;166(1):319-27.

27. Sundara SB, Seol E, Mohan RS, Park S. Co-production of hydrogen and ethanol by pfkA-deficient Escherichia Coli with activated pentosephosphate pathway: reduction of pyruvate accumulation. Biotechnol Biofuels. 2016;9(1):1-11.

28. Okochi M, Kurimoto M, Shimizu K, Honda H. Increase of organic solvent tolerance by overexpression of manXYZ in Escherichia Coli. Appl Microbiol Biot. 2007;73(6):1394-9.

29. Ohta K, Hamasuna H, Tsukamoto J, Wakiyama M, Izumi Y, Harada K. Disruption of ptsG gene and manXYZ operon of ethanol-producing Escherichia Coli KO11: effects on glucose and xylose utilization and ethanol production. J Biosci Bioeng. 2012;113(5):608-10.

30. Leach S, Harvey P, Wali R. Changes with growth rate in the membrane lipid composition of and amino acid utilization by continuous cultures of campylobacter jejuni. J Appl Microbiol. 1997;82(5):631-40.

31. Velayudhan J, Jones MA, Barrow PA, Kelly DJ. L-serine catabolism via an oxygen-labile L-serine Dehydratase is essential for colonization of the avian gut by campylobacter jejuni. Infect Immun. 2004;72(1):260-8.

32. Takasu A, Makino T, Hirabayashi T. From the regulation of PG synthesis to bacterial growth and morphology. Nat Rev Microbiol. 2011;10(2):123-36.

33. Bearne SL, Blouin C. Inhibition of Escherichia coliGlucosamine-6-phosphate Synthase by reactive intermediate analogues. J Biol Chem. 2003;275(1):135-40.

34. Höltje JV, Tuomanen El. The murein hydrolases of Escherichia coli: properties, functions and impact on the course of infections in vivo. J of Gen Microbiol. 1991;137(3):441-54.

35. Jazaeri S, Amiri-Simkooei A, Sharifi MA. The humoral pattern recognition receptor PTX3 is stored in neutrophil granules and localizes in extracellular traps. J Exp Med. 2007;204(4):793-804

36. Doni A, Michela M, Bottazzi B, Peri G, Valentino S, Polentarutti N, et al. Regulation of PTX3, a key component of humoral innate immunity in human dendritic cells: stimulation by IL-10 and inhibition by IFN-gamma. J Leukoc Biol. 2006;79(4):797-802

37. Zganiacz A, Santosuosso M, Wang J, Yang T, Chen L, Anzulovic M, et al. TNF-a is a critical negative regulator of type 1 immune activation during intracellular bacterial infection. J Clin Invest. 2004;113(3):401-13.

38. Nagabhushanam V, Solache A, Ting LM, Escaron CJ, Zhang JY, Ernst JD. Innate inhibition of adaptive immunity: mycobacterium tuberculosisinduced IL-6 inhibits macrophage responses to IFN-gamma. J Immunol. 2003:171(9):4750-7.

39. Bendelac A, Savage PB, Teyton L. The biology of NKT cells. Immunology 2007:25(25):297-336

40. Doisne JM, Soulard V, Bécourt C, Amniai L, Henrot P, Havenardaughton C, et al. Cutting edge: crucial role of IL-1 and IL-23 in the innate IL-17 response of peripheral lymph node NK1.1- Invariant NKT cells to bacteria. J Immunol. 2011;186(2):662-6.

41. Iwakura $Y$, Ishigame $H$. The IL-23/IL-17 axis in inflammation. J Clin Invest. 2006;116(5):1218-22.

42. Yen D, Cheung J, Scheerens H, Poulet F, Mcclanahan T, Mckenzie B, et al. IL-23 is essential for T cell-mediated colitis and promotes inflammation via IL-17 and IL-6. J Clin Invest. 2006;116(5):1310-6.

43. Gutcher I, Donkor MK, Ma Q, Rudensky AY, Flavell RA, Li MO. Autocrine transforming growth factor- $\beta 1$ promotes in vivo Th17 cell differentiation. Immunity. 2011;34(3):396-408.

44. Chung Y, Chang SH, Martinez GJ, Yang XO, Nurieva R, Kang HS, et al. Critical regulation of early Th17 cell differentiation by Interleukin-1 signaling. Immunity. 2009;30(4):576-87.

45. Ivanov II, Zhou L, Littman DR. Transcriptional regulation of Th17 cell differentiation. Semin Immunol. 2007;19(6):409-17.

46. Segura E, Touzot M, Bohineust A, Cappuccio A, Chiocchia G, Hosmalin A, et al. Human inflammatory Dendritic cells induce Th17 cell differentiation. Immunity. 2013;8(2):336-48.
47. Kimura A, Kishimoto T. IL-6: regulator of Treg/Th17 balance. Eur J Immunol. 2010;40(7):1830-5.

48. Ouyang W, Valdez P. IL-22 in mucosal immunity. Mucosal Immunol. 2008;1(5):335-8.

49. Feinen B, Russell MW. Contrasting roles of IL-22 and IL-17 in Murine genital tract infection by Neisseria gonorrhoeae. Front Immuno. 2012;3:11. https:// doi.org/10.3389/fimmu.2012.00011.

50. Zheng Y, Valdez PA, Danilenko DM, Hu Y, Sa SM, Gong Q, et al. Interleukin22 mediates early host defense against attaching and effacing bacterial pathogens. Nat Med. 2008;14(3):282-9.

51. Aujla SJ, Chan YR, Zheng M, Fei M, Askew DJ, Pociask DA, et al. IL-22 mediates mucosal host defense against gram-negative bacterial pneumonia. Nat Med. 2008;14(3):275-81.

52. Ye P, Rodriguez FH, Kanaly S, Stocking KL, Schurr J, Schwarzenberger P, et al. Requirement of interleukin 17 receptor signaling for lung Cxc Chemokine and granulocyte Colony-stimulating factor expression, Neutrophil recruitment, and host defense. J Exp Med. 2001:194(4):519-27.

53. Goldblum SE, Hennig B, Jay M, Yoneda K, Mcclain CJ. Tumor necrosis factor alpha-induced pulmonary vascular endothelial injury. Infect Immun. 1989;57(4):1218-26.

54. Zobel K, Martus P, Pletz MW, Ewig S, Prediger M, Welte $T$, et al. Interleukin 6, lipopolysaccharide-binding protein and interleukin $10 \mathrm{in}$ the prediction of risk and etiologic patterns in patients with community-acquired pneumonia: results from the German competence network CAPNETZ. BMC Pulm Med. 2012;12(1):1-10.

55. Rodriguez N, Dietrich H, Mossbrugger I, Weintz G, Scheller J, Hammer M, et al. Increased inflammation and impaired resistance to Chlamydophila pneumoniae infection in Dusp1(-/-) mice: critical role of IL-6. J Leukoc Biol. 2010;88(3):579-87.

56. Baltes N, Hennigpauka I, Jacobsen I, Gruber AD, Gerlach GF. Identification of Dimethyl Sulfoxide Reductase in Actinobacillus pleuropneumoniae and its role in infection. Infect Immun. 2004;71(12):6784-92.

57. Ilse J, Isabel H-P, Nina B, et al. Enzymes involved in anaerobic respiration appear to play a role in Actinobacillus pleuropneumoniae virulence. Infect Immun. 2005;73(1):226-34.

58. Baltes N, N'Diaye M, Jacobsen ID, Maas A, Buettner FF, Gerlach GF. Deletion of the anaerobic regulator HlyX causes reduced colonization and persistence of Actinobacillus pleuropneumoniae in the porcine respiratory tract. Infect Immun. 2005;73(8):4614-9.

59. Baltes N, Buettner FFR, Gerlach GF. Selective capture of transcribed sequences (SCOTS) of Actinobacillus pleuropneumoniae in the chronic stage of disease reveals an HlyX-regulated autotransporter protein. Vet Microbiol. 2007;123(1-3):110-21.

60. Clark D, Cronan JE Jr. Escherichia Coli mutants with altered control of alcohol dehydrogenase and nitrate reductase. J Bacteriol. 1980;141(1):177-83.

61. Leonardo MR, Dailly Y, Clark DP. Role of NAD in regulating the adhE gene of Escherichia Coli. J Bacteriol. 1996;178(20):6013-8.

62. Beckham KSH, Connolly JPR, Ritchie JM, Wang D, Gawthorne JA, Tahoun A, et al. The metabolic enzyme AdhE controls the virulence of $E$ scherichia coli O157:H7. Mol Microbiol. 2014;93(1):199-211.

63. Echave P, Tamarit J, Cabiscol E, Ros J. Novel antioxidant role of alcohol dehydrogenase E from Escherichia coli. J Biol Chem. 2003;278(32):30193-8.

64. Vollmer W, Bertsche U. Murein (peptidoglycan) structure, architecture and biosynthesis in Escherichia Coli. Biochim Biophys Acta. 2008;1778(9):1714-34

65. Bishop JL, Finlay BB. Deception point: peptidoglycan modification as a means of immune evasion. Proc Natl Acad Sci U S A. 2007;104(3):691-2.

66. Boneca IG, Dussurget $O$, Cabanes D, Nahori MA, Sousa S, Lecuit M, et al. A critical role for peptidoglycan N-deacetylation in Listeria evasion from the host innate immune system. Proc Natl Acad Sci U S A. 2007:104(3):997-1002.

67. Suzuki M, Miura S, Suematsu M, Fukumura D, Kurose I, Suzuki H, et al. Helicobacter pylori-associated ammonia production enhances neutrophil-dependent gastric mucosal cell injury. Am J Phys. 1992; 263(1):719-25.

68. Bossé JT, Macinnes Jl. Genetic and biochemical analyses of Actinobacillus pleuropneumoniae urease. Infect Immun. 1997:65(11):4389-94.

69. Rioux S, Galarneau C, Harel J, Kobisch M, Frey J, Gottschalk M, et al. Isolation and characterization of a capsule-deficient mutant of Actinobacillus pleuropneumoniae serotype 1. Microb Pathog. 2000;28(5):279-89. 
70. Langmead B, Trapnell C, Pop M, Salzberg SL. Ultrafast and memory-efficient alignment of short DNA sequences to the human genome. Genome Biol. 2009;10(3):1-10.

71. Kim D, Pertea G, Trapnell C, Pimentel H, Kelley R, Salzberg SL. TopHat2: Accurate alignment of transcriptomes in the presence of insertions, deletions and gene fusions. Genome Biol. 2013;14(4):R36. https://doi.org/10. 1186/gb-2013-14-4-r36.

72. Jiang $H$, Wong WH. Statistical inferences for isoform expression in RNA-Seq. Bioinformatics. 2009;25(8):1026-32.

73. Djebali S, Davis CA, Merkel A, Dobin A, Lassmann T, Mortazavi A, et al. Landscape of transcription in human cells. Nature. 2012:489(7414):101-8.

74. Leng N. EBSeq: an empirical Bayes hierarchical model for inference in RNAseq experiments. Bioinformatics. 2013;29(8):1035-43.

Submit your next manuscript to BioMed Central and we will help you at every step:

- We accept pre-submission inquiries

- Our selector tool helps you to find the most relevant journal

- We provide round the clock customer support

- Convenient online submission

- Thorough peer review

- Inclusion in PubMed and all major indexing services

- Maximum visibility for your research

Submit your manuscript at www.biomedcentral.com/submit
Biomed Central 